\title{
Combination of Aromatase Inhibitors with Metronomic Capecitabine: A New Chemoendocrine Treatment for Advanced Breast Cancer
}

\author{
Wei Shi, Xinyue Wang, Xiwen Bi, Wen Xia, Jiajia Huang, Yanhong Su, Zhangzan Huang, \\ Zhongyu Yuan*
}

Department of Medical Oncology, Sun Yat-sen University Cancer Center, State Key Laboratory of Oncology in South China, Collaborative Innovation Center for Cancer Medicine, Guangzhou, China

Email: *yuanzhy@sysucc.org.cn

How to cite this paper: Shi, W., Wang, X.Y., Bi, X.W., Xia, W., Huang, J.J., Su, Y.H., Huang, Z.Z. and Yuan, Z.Y. (2019) Combination of Aromatase Inhibitors with Metronomic Capecitabine: A New Chemoendocrine Treatment for Advanced Breast Cancer. Journal of Cancer Therapy, 10, 146-156.

https://doi.org/10.4236/jct.2019.102011

Received: January 7, 2019

Accepted: February 12, 2019

Published: February 15, 2019

Copyright $\odot 2019$ by author(s) and Scientific Research Publishing Inc. This work is licensed under the Creative Commons Attribution International License (CC BY 4.0).

http://creativecommons.org/licenses/by/4.0/

\begin{abstract}
Purpose: Endocrine therapy is one of the main treatment options for hormone receptor (HR)-positive advanced breast cancer (ABC). However, whether the combination of endocrine therapy with chemotherapy is practicable and more effective than endocrine therapy alone remains unknown. The aim of this study was to investigate the clinical efficacy of the aromatase inhibitors (AIs) combined with metronomic capecitabine to provide the clinical evidence for further research in patients with HR-positive ABC. Methods: Data from 407 patients with HR-positive $\mathrm{ABC}$ were retrospectively analyzed. A total of 305 patients were given AIs alone, and 102 patients were given AIs plus capecitabine as first-line treatment. Progression-free survival (PFS) was the primary endpoint. Results: The median follow-up for all patients was 47.0 months (range, 3 - 119 months). The median overall survival (OS) and PFS were 52.0 months and 24.2 months, respectively. The median PFS in the combination group was significantly longer than that in the AIs group (22.0 months vs. 14.0 months, $p=0.002$ ). Additionally, patients in the combination group had significantly longer OS than patients in the AI group (66.0 months vs. 49.0 months, $p=0.003$ ). Multivariate analysis showed that combination therapy was a significant favorable predictor for PFS and OS. Furthermore, young age ( $<40$ years), low estrogen receptor (ER) expression level $(<40 \%)$, presence of visceral metastasis, prior adjuvant AI use and long disease-free interval (DFI) (>24 months) improved the benefit from combination therapy. Conclusions: AIs plus metronomic capecitabine significantly improves PFS and OS in patients with HR-positive ABC. Thus, chemo-endocrine therapy
\end{abstract}


should be further explored.

\section{Keywords}

Advanced Breast Cancer, HR, Edocrine Resistance, Chemoendocrine Therapy, Survival

\section{Introduction}

Breast cancer is the most common cause of cancer death in women [1]. Approximately $20 \%$ to $30 \%$ patients with early-stage breast cancer will eventually progress to metastatic disease [2], and $6 \%$ to $10 \%$ of newly diagnosed breast cancer cases are metastatic [3]. Except for cases with visceral metastasis, larger tumor burden and rapid growing tumors, guidelines recommend endocrine therapy as the preferred treatment option for hormone receptor (HR)-positive advanced breast cancer (ABC) [4] [5]. Although endocrine therapy has shown good efficacy and tolerance in both early- and advanced-stage disease, drug resistance is inevitable. Substantial efforts have been recently made to overcome resistance to endocrine therapy. New endocrine therapies, such as estrogen receptor (ER) degraders (fulvestrant), which directly bind to HRs, have been developed [6]. In addition, mTOR inhibitors and CDK4/6 inhibitors, which target the bypass pathways activated in the mechanisms of endocrine treatment resistance, have greatly improved progression-free survival (PFS) and overall survival (OS) in patient with ABC [7] [8]. Precision and targeted treatments are being developed against endocrine treatment resistance. However, some patients experiencing disease progression after endocrine therapy may actually have pseudoresistance (Figure 1). In other words, these patients experience disease progression following endocrine therapy not because of resistance but because of the proliferation of HR-negative cells over time. Metronomic chemotherapy (MC) as a new regime of low-dose and continuous chemotherapy is efficient and well tolerated [9]. We thus speculate that metronomic chemotherapy combined with endocrine therapy to target different cells in HR-positive $\mathrm{ABC}$ may be more effective than endocrine therapy alone.

Decades ago, a number of studies had suggested that the combination of tamoxifen and chemotherapy not only reduced efficacy but also led to additional toxicity [10] [11] [12]. Therefore, at that time, a consensus was reached that endocrine therapy should not be used in combination with chemotherapy. However, with the development of new generations of endocrine treatments, the situation has been reversed. In vitro and in vivo experiments suggest that combination of aromatase inhibitors (AIs) or fulvestrant with chemotherapy exerts a synergistic effect on the suppression of tumor growth [13] [14] [15].

Recently, data from clinical trials demonstrated a therapeutic potential for the combination of AIs or fulvestrant with chemotherapy in HR-positive ABC and combination of therapy, especially capecitabinemetronomic chemotherapy, was 


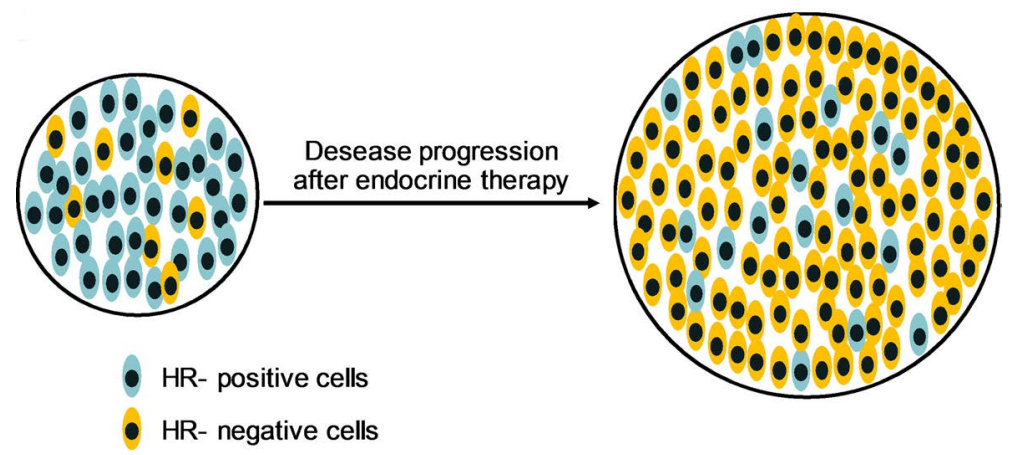

Figure 1. HR-negative cells proliferate after endocrine therapy.

well tolerated and efficient [16] [17] [18]. Based on existing evidence, it is reasonable to assume that the combination of new-generation endocrine therapy and metronomic chemotherapy may be a good choice for HR-positive ABC.

The aim of this retrospective study was to evaluate the clinical efficacy of a synchronous therapy, metronomic chemotherapy with oral capecitabine in combination with third-generation AIs for HR-positive $\mathrm{ABC}$ to provide the clinical evidence for further research.

\section{Patients and Methods}

\subsection{Study Population}

The eligible patients were diagnosed with HR-positive and human epidermal growth factor receptor type 2 (HER2)-negative invasive breast cancer and treated at Sun Yat-sen University Cancer Center from January 2012 to December 2015. Patients were included based on the following criteria: 1) metastatic breast cancer; 2) histological confirmation of invasive breast cancer; 3) ERand/or progesterone receptor (PR)-positive, HER2-negative advanced cancer; 4) availability of complete medical records and follow-up status; 5) no prior treatment after diagnosis of metastatic breast cancer. A total of 407 patients with $\mathrm{ABC}$ were recruited in this study. The study was reviewed and approved by the Institutional Review Board of Sun Yat-sen University Cancer Center.

\subsection{Treatments and Evaluation}

Of the $407 \mathrm{ABC}$ patients, 305 were treated with AIs alone, and 102 were treated with AIs plus metronomic capecitabine as first-line treatment. AIs included letrozole, anastrozole or exemestane. The dosage was respectively $10 \mathrm{mg}, 1 \mathrm{mg}$, and $25 \mathrm{mg}$ once daily. Capecitabine was administered at $500 \mathrm{mg} 3$ times daily. In addition, all premenopausal patients were under ovarian suppression, including monthly hormone injections or surgery to remove the ovaries. Treatment continued until disease progression or the emergence of intolerable toxicity. The reduction of the capecitabine dose was permitted: an initial reduction to $500 \mathrm{mg}$ twice daily and a subsequent reduction to $500 \mathrm{mg}$ once daily if 2 degrees or above toxicity according to CTCAE 4.1. AIs were not applied with reduction of 
doses. Response to treatment was evaluated by computed tomography or magnetic resonance imaging every 2 months until disease progression according to the response evaluation criteria in solid tumors [19].

\subsection{Statistical Analyses}

The primary endpoint of this study was PFS, defined as the time from the beginning of treatment for metastatic breast cancer to disease progression or death from any cause. The secondary endpoint was OS, defined as the period from the date of treatment for metastatic breast cancer to the date of death from any cause or the date of last follow-up. Clinicopathologic parameters were assessed between the two groups by chi-square test. The median PFS and OS were calculated by using the Kaplan-Meier method. Survival rates were compared by log-rank test. Multivariate analyses were performed using the Cox regression model for PFS and OS to identify independent factors and adjust for baseline characteristics. All analyses were performed using SPSS for Windows version 20.0 (IBM Corp. Armonk, USA). All statistical tests were two-sided, and $p$ values $<0.05$ were considered significant.

The authenticity of this article has been validated by uploading the key raw data onto the Research Data Deposit public platform (http://www.researchdata.org.cn), with the approval RDD number as RDDA2018000599.

\section{Results}

\subsection{Patient Characteristics and Treatment}

The clinicopathologic characteristics of the 407 patients in this study are described in Table 1. The median age was 45 years (range, 21 - 72 years) in the AI group and 44 years (range, 24 - 75 years) in the combination group. The number of patients with ER levels $\leq 40 \%, 40 \%-70 \%$ and $\geq 70 \%$, visceral metastasis or disease-free interval (DFI) $>24$ months was not significantly different between the two groups. A higher proportion of patients in the combination group used adjuvant AIs than that in the AI group ( $70.6 \%$ vs. $53.4 \%, p=0.002)$.

\subsection{PFS and OS}

The median follow-up for all patients was 47.0 months (range, 3 - 119 months). During the follow-up period, a total of 305 patients died, including 236 (77.4\%) patients in the AI group and 69 (22.6\%) patients in the combination group. The 4-year PFS rates in the AI and combination groups were $10.8 \%$ and $23.2 \%$, respectively. The 4 -year OS rates in the AI and combination groups were $52.0 \%$ and $63.3 \%$, respectively.

The median OS and PFS for all patients were 52.0 months and 14.0 months, respectively. The median PFS in the AI group was significantly shorter than that in the combination group (14.0 months vs. 22.0 months, $p=0.002)$. Additionally, the median OS in the AI group was significantly shorter than that in the combination group (49.0 months vs. 66.0 months, $p=0.003$ ) (Figure 2). 
Table 1. Clinical characteristics and treatment of breast cancer patients.

\begin{tabular}{cccc}
\hline Characteristics & AIs group & Combination group & \multirow{2}{*}{$p$ value } \\
\cline { 2 - 3 } Age (years) & $\mathrm{N}(\%)$ & $\mathrm{N}(\%)$ & \\
$\leq 40$ & $189(62.0 \%)$ & $61(59.8 \%)$ & 0.725 \\
$>40$ & $116(38.0 \%)$ & $41(40.2 \%)$ & \\
ER level & & & \multirow{2}{*}{0.078} \\
$\leq 40 \%$ & $115(50.2 \%)$ & $50(49.0 \%)$ & \\
$40 \%$ - 70\% & $111(36.4 \%)$ & $30(29.4 \%)$ & 0.754 \\
$\geq 70 \%$ & $41(13.4 \%)$ & $22(21.6 \%)$ & \\
Visceral metastasis & & & \\
No & $134(43.9 \%)$ & $43(42.2 \%)$ & $0.002^{*}$ \\
Yes & $171(56.1 \%)$ & $59(57.8 \%)$ & \\
Adjuvant AIs & & & \\
No & $142(46.6 \%)$ & $30(29.4 \%)$ & 0.514 \\
Yes & $163(53.4 \%)$ & $72(70.6 \%)$ & \\
Disease-free interval & & & \\
$\leq 24$ months & $173(64.4 \%)$ & $53(52.0 \%)$ & \\
$>24$ months metastasis & $132(35.6 \%)$ & $49(48.0 \%)$ & \\
Liver & $57(18.7 \%)$ & $16(15.7 \%)$ & \\
Lung & $65(21.2 \%)$ & $35(34.3 \%)$ & \\
Bone & $187(61.3 \%)$ & $65(63.7 \%)$ & \\
Local recurrence & $89(29.2 \%)$ & $37.2 \%)$ & \\
\hline
\end{tabular}

ER: estrogen receptor; AIs: aromatase inhibitors. Statistical analysis was performed using $\chi^{2}$ test. Statistically significant differences are labeled*.
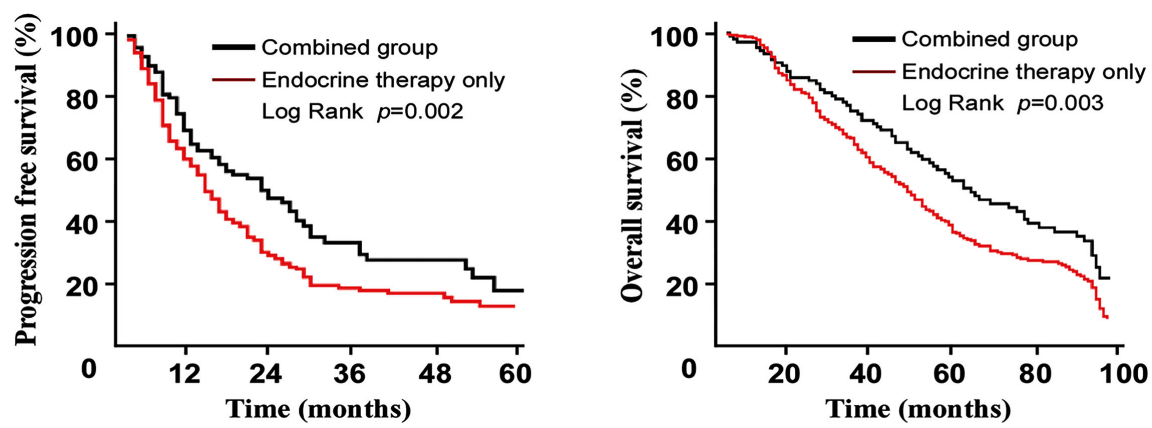

Figure 2. The PFS and OS in the combination group were significantly longer than that in the endocrine therapy group.

\subsection{Effects of Prognostic Factors on PFS and OS}

To evaluate the independent role of various variables in PFS and OS, we performed a multivariate analysis including age, ER level, visceral metastasis, adjuvant $\mathrm{AI}$ use, DFI and combination therapy. The presence of visceral metastasis was an adverse prognostic factor for PFS and OS. The combination of AIs with capecitabine was a favorable prognostic factor for PFS and OS. Moreover, lower ER expression level $(<40 \%)$, presence of visceral metastasis, relapse following adjuvant AIs and shorter DFI were adverse prognostic factors for OS but not for PFS (Table 2). 
To explore the benefit from combination therapy, we performed univariate analysis, which showed that lower ER expression level $(<40 \%)$, presence of visceral metastasis, prior adjuvant AI use and longer DFI ( $>24$ months) significantly improved the benefit from combination therapy (Figure 3 ).

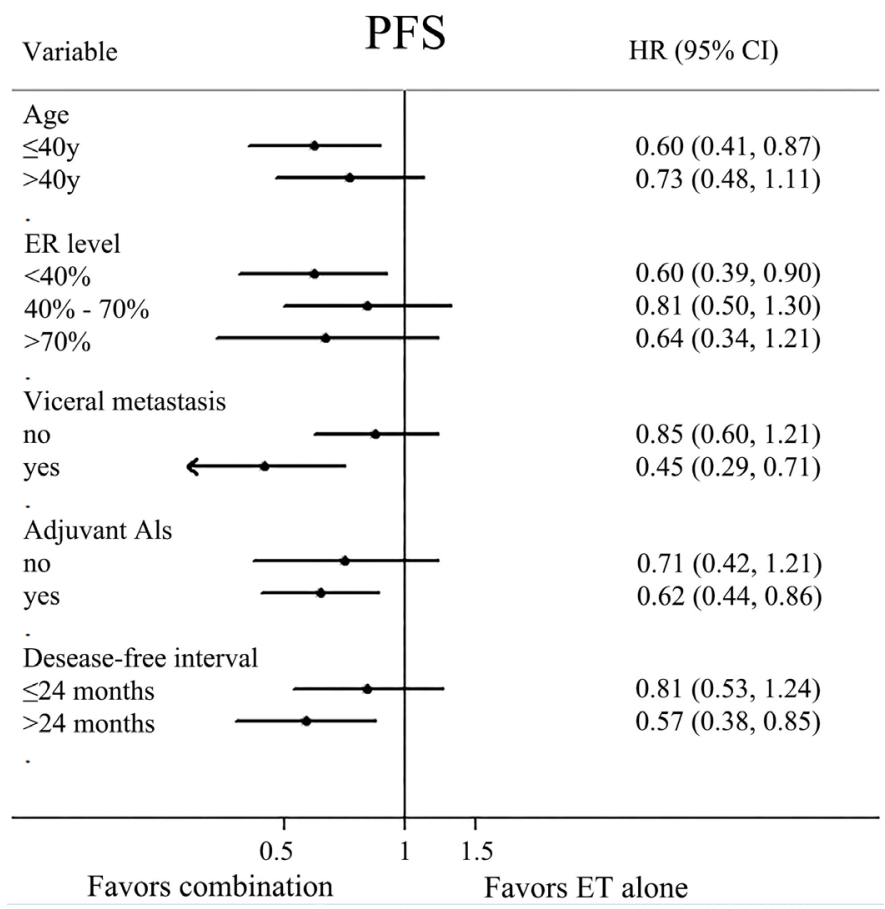

(a)

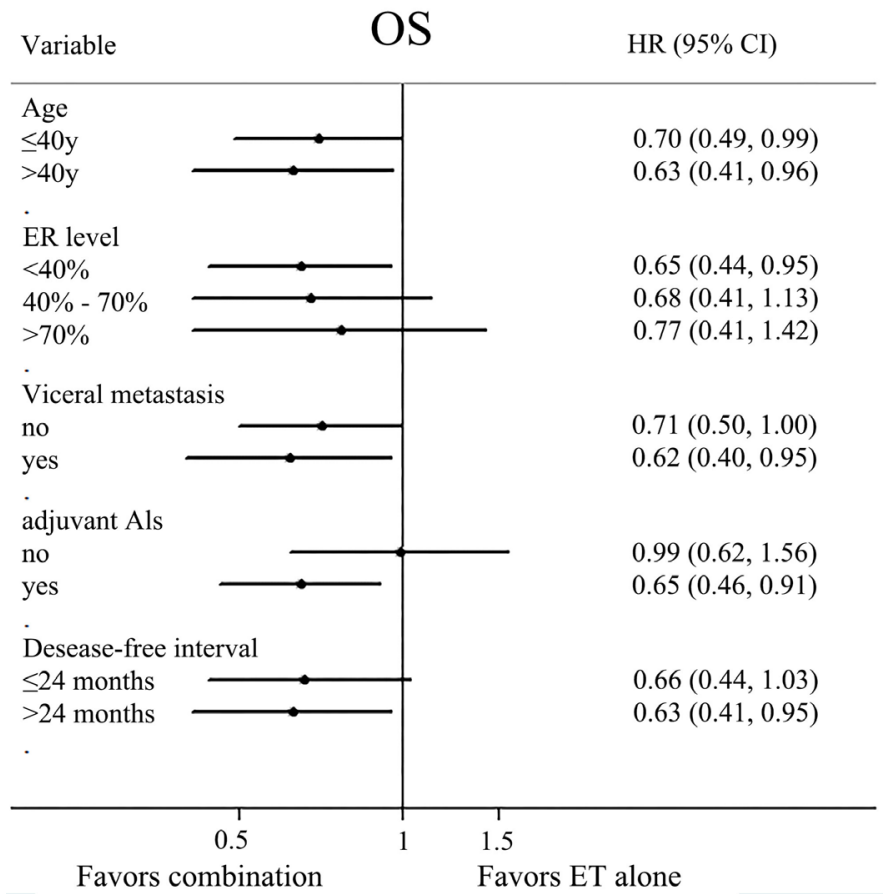

(b)

Figure 3. Lower ER expression level, visceral metastasis, prior adjuvant AI use and longer DFI improved the benefit from combination therapy. 
Table 2. Multivariate analysis of PFS and OS in all population.

\begin{tabular}{ccccccc}
\hline \multirow{2}{*}{ Variables } & \multicolumn{4}{c}{ PFS } & \multicolumn{3}{c}{ OS } \\
\cline { 2 - 7 } & HR & $95 \%$ CI & $p$ value & HR & $95 \%$ CI & $p$ value \\
\hline Age, years $(\leq 40$ vs. $>40)$ & 0.94 & $0.74-1.20$ & 0.627 & 1.01 & $0.79-1.28$ & 0.960 \\
ER level (<40\% vs. $\geq 40 \%)$ & 0.98 & $0.74-1.30$ & 0.895 & 0.43 & $0.32-0.59$ & $<0.001^{*}$ \\
Visceral metastasis (no vs. yes) & 1.29 & $1.02-1.63$ & $0.035^{\star}$ & 1.54 & $1.22-1.95$ & $<0.001^{*}$ \\
Adjuvant AIs (no vs. yes) & 0.91 & $0.67-1.23$ & 0.540 & 2.71 & $2.00-3.67$ & $<0.001^{*}$ \\
DFI ( $\leq 24$ months vs. $>24$ months) & 0.97 & $0.75-1.24$ & 0.781 & 0.34 & $0.27-0.44$ & $<0.001^{*}$ \\
AIs plus Cap. (no vs. yes) & 0.65 & $0.49-0.87$ & $0.003^{\star}$ & 0.72 & $0.55-0.95$ & $0.020^{*}$ \\
\hline
\end{tabular}

ER: estrogen receptor; AIs: aromatase inhibitors; Cap: capecitabine; HR: hazard ratio; HR and 95\% CIs were calculated using COX regression analysis. Statistically significant differences are labeled ${ }^{*}$. DFI: disease-free interval.

\section{Discussion}

Endocrine therapy is generally the first treatment option for HR-positive ABC. The PFS of patients treated with first-line tamoxifen, AIs and fulvestrant is approximately 6 months, 9 - 13 months and 17 - 23 months, respectively [20] [21] [22] [23] [24]. In other words, all patients inevitably experience disease progression. Various mechanisms, either intrinsic or acquired, could be implicated in endocrine therapy resistance [25]. However, in some patients, disease progression following endocrine therapy may not be because of endocrine resistance but rather due to the proliferation of some HR-negative cancer cells during the period of endocrine therapy. In some cases, ten percent of cells can be HR-negative, even though $90 \%$ of cells are HR-positive. Therefore, endocrine therapy alone is not sufficient to suppress the growth of so called "HR-positive" breast cancer. Additionally, in this study, we are interested in the theoretical basis of chemo-endocrine therapy.

The results of some preclinical studies on the efficacy of chemo-endocrine therapy are still uncertain [10] [14] [15] [26] [27] [28] [29]. In the past, it had been proposed that tamoxifen induced the accumulation of breast cancer cells in the G0/G1 phase and that cells in this phase of the cell cycle were not sensitive to chemotherapy (epirubicin plus cyclophosphamide) [10]. The combined use of chemotherapy and tamoxifen in early-stage breast cancer is not realistic [11] [30] and is not recommended by guidelines. However, AIs and fulvestrant are currently widely used for HR-positive breast cancer. They have different mechanisms of action and superior efficacy in comparison with tamoxifen. Several clinical trials also show that AIs or fulvestrant plus intravenous or oral chemotherapies result in high overall response rate or prolonged PFS in patients with HR-positive ABC [16] [18] [31]. These results show that the combination of endocrine therapy with metronomic chemotherapy may be a rational option without any antagonistic effect.

Our results suggested that compared with AIs alone, AIs plus metronomic capecitabine as first-line therapy significantly improved PFS and OS in patients 
with $\mathrm{ABC}$. In particular, patients with low ER expression, visceral metastasis, prior adjuvant AI therapy and prolonged DFI displayed obvious benefits from chemo-endocrine therapy. Patients with lower ER expression level had a lower response to endocrine therapy [32]. The presence of visceral metastasis and short DFI suggests an unfavorable prognosis [33]. Response to AIs following adjuvant AIs may be significantly lower than that to treatments without prior adjuvant AIs [22] [34]. In brief, patients with HR-positive $\mathrm{ABC}$ with unfavorable prognostic factors or patients who are insensitive to endocrine therapy may be candidates for chemo-endocrine therapy.

Nevertheless, we recognize there are several limitations to this study. First, this study was a retrospective analysis. Second, selective bias might be inevitable (e.g., the combination group had a higher proportion of patients who received adjuvant AIs that did the AI group, and the reason might be that clinicians were more likely to use combination therapy if patients had already received adjuvant AIs). Despite these limitations, our results demonstrate that the combination of AIs with metronomic capecitabine significantly improves PFS and OS in patients with HR-positive ABC. Chemo-endocrine therapy may thus be considered a therapeutic strategy for these patients. We are currently conducting a larger randomized controlled phase 3 trial to support our findings (NCT02767661).

\section{Ethical Approval}

All procedures performed in studies involving human participants were in accordance with the ethical standards of the institutional and/or national research committee and with the 1964 Helsinki declaration and its later amendments or comparable ethical standards.

\section{Conflicts of Interest}

The authors declare no conflicts of interest regarding the publication of this paper.

\section{References}

[1] Torre, L.A., Bray, F., Siegel, R.L., Ferlay, J., Lortet-Tieulent, J. and Jemal, A. (2015) Global Cancer Statistics, 2012. CA: A Cancer Journal for Clinicians, 65, 87-108. https://doi.org/10.3322/caac.21262

[2] O’Shaughnessy, J. (2005) Extending Survival with Chemotherapy in Metastatic Breast Cancer. The Oncologist, 10, 20-29. https://doi.org/10.1634/theoncologist.10-90003-20

[3] Metastatic Breast Cancer Network (2016) Incidence and Incidence Rates. http://www.mbcn.org/incidence-and-incidence-rates/

[4] Cardoso, F., Costa, A., Norton, L., Senkus, E., Aapro, M., Andre, F., et al. (2014) ESO-ESMO 2nd International Consensus Guidelines for Advanced Breast Cancer (ABC2) Dagger. Annals of Oncology, 25, 1871-1888. https://doi.org/10.1093/annonc/mdu385

[5] Gradishar, W.J., Anderson, B.O., Balassanian, R., Blair, S.L., Burstein, H.J., Cyr, A., et al. (2017) NCCN Guidelines Insights: Breast Cancer, Version 1.2017. Journal of 
the National Comprehensive Cancer Network. JNCCN, 15, 433-451.

https://doi.org/10.6004/jnccn.2017.0044

[6] Di Leo, A., Jerusalem, G., Petruzelka, L., Torres, R., Bondarenko, I.N., Khasanov, R., et al. (2010) Results of the CONFIRM Phase III Trial Comparing Fulvestrant 250 mg with Fulvestrant $500 \mathrm{mg}$ in Postmenopausal Women with Estrogen Receptor-Positive Advanced Breast Cancer. Journal of Clinical Oncology, 28, 4594-4600. https://doi.org/10.1200/JCO.2010.28.8415

[7] Yardley, D.A., Noguchi, S., Pritchard, K.I., Burris III, H.A., Baselga, J., Gnant, M., et al. (2013) Everolimus Plus Exemestane in Postmenopausal Patients with HR(+) Breast Cancer: BOLERO-2 Final Progression-Free Survival Analysis. Advances in therapy, 30, 870-884. https://doi.org/10.1007/s12325-013-0060-1

[8] Finn, R.S., Crown, J.P., Lang, I., Boer, K., Bondarenko, I.M., Kulyk, S.O., et al. (2015) The Cyclin-Dependent Kinase 4/6 Inhibitor Palbociclib in Combination with Letrozole Versus Letrozole Alone as First-Line Treatment of Oestrogen Receptor-Positive, HER2-Negative, Advanced Breast Cancer (PALOMA-1/TRIO-18): A Randomised Phase 2 Study. The Lancet Oncology, 16, 25-35. https://doi.org/10.1016/S1470-2045(14)71159-3

[9] Montagna, E., Cancello, G., Dellapasqua, S., Munzone, E. and Colleoni, M. (2014) Metronomic Therapy and Breast Cancer: A Systematic Review. Cancer Treatment Reviews, 40, 942-950. https://doi.org/10.1016/j.ctrv.2014.06.002

[10] Hug, V.H.G., Drewinko, B. and Finders, M. (1985) Tamoxifen-Citrate Counteracts the Antitumor Effects of Cytotoxic Drugs in Vitro. Journal of Clinical Oncology, 3, 1672-1677. https://doi.org/10.1200/JCO.1985.3.12.1672

[11] Albain, K.S., Barlow, W.E., Ravdin, P.M., Farrar, W.B., Burton, G.V., Ketchel, S.J., et al. (2009) Adjuvant Chemotherapy and Timing of Tamoxifen in Postmenopausal Patients with Endocrine-Responsive, Node-Positive Breast Cancer: A Phase 3, Open-Label, Randomised Controlled Trial. The Lancet, 374, 2055-2063.

https://doi.org/10.1016/S0140-6736(09)61523-3

[12] Pico, C., Martin, M., Jara, C., Barnadas, A., Pelegri, A., Balil, A., et al. (2004) Epirubicin-Cyclophosphamide Adjuvant Chemotherapy plus Tamoxifen Administered Concurrently versus Sequentially: Randomized Phase III Trial in Postmenopausal Node-Positive Breast Cancer Patients. A GEICAM 9401 Study. Annals of Oncology, 15, 79-87. https://doi.org/10.1093/annonc/mdh016

[13] Sui, M.H., Jiang, D.H., Hinsch, C. and Fan, W.M. (2010) Fulvestrant (ICI 182,780) Sensitizes Breast Cancer Cells Expressing Estrogen Receptor Alpha to Vinblastine and Vinorelbine. Breast Cancer Research and Treatment, 121, 335-345. https://doi.org/10.1007/s10549-009-0472-4

[14] Ikeda, H., Taira, N., Nogami, T., Shien, K., Okada, M., Shien, T., et al. (2011) Combination Treatment with Fulvestrant and Various Cytotoxic Agents (Doxorubicin, Paclitaxel, Docetaxel, Vinorelbine, and 5-Fluorouracil) Has a Synergistic Effect in Estrogen Receptor-Positive Breast Cancer. Cancer Science, 102, 2038-2042. https://doi.org/10.1111/j.1349-7006.2011.02050.x

[15] Kataoka, M., Yamaguchi, Y., Moriya, Y., Sawada, N., Yasuno, H., Kondoh, K., et al. (2012) Antitumor Activity of Chemoendocrine Therapy in Premenopausal and Postmenopausal Models with Human Breast Cancer Xenografts. Oncology Reports, 27, 303-310. https://doi.org/10.3892/or.2011.1541

[16] de la Haba-Rodriguez, J., Mancha, R.G., Manga, G.P., Aguilar, E.A., Baena Canada, J.M., Rovira, P.S., et al. (2010) Exemestane and Chemotherapy as First-Line Treatment of Metastatic Breast Cancer: Results of a Phase II Study. Clinical Breast Cancer, 10, 313-317. https://doi.org/10.3816/CBC.2010.n.041 
[17] Aurilio, G., Munzone, E., Botteri, E., Sciandivasci, A., Adamoli, L., Minchella, I., et al. (2012) Oral Metronomic Cyclophosphamide and Methotrexate plus Fulvestrant in Advanced Breast Cancer Patients: A Mono-Institutional Case-Cohort Report. The Breast Journal, 18, 470-474. https://doi.org/10.1111/j.1524-4741.2012.01278.x

[18] Schwartzberg, L.S., Wang, G., Somer, B.G., Blakely, L.J., Wheeler, B.M., Walker, M.S., et al. (2014) Phase II Trial of Fulvestrant with Metronomic Capecitabine for Postmenopausal Women with Hormone Receptor-Positive, HER2-Negative Metastatic Breast Cancer. Clinical Breast Cancer, 14, 13-19.

https://doi.org/10.1016/j.clbc.2013.09.003

[19] Eisenhauer, E.A., Therasse, P., Bogaerts, J., Schwartz, L.H., Sargent, D., Ford, R., et al. (2009) New Response Evaluation Criteria in Solid Tumours: Revised RECIST Guideline (Version 1.1). European Journal of Cancer, 45, 228-247. https://doi.org/10.1016/j.ejca.2008.10.026

[20] Bonneterre, J., Thurlimann, B., Robertson, J.F., Krzakowski, M., Mauriac, L., Koralewski, P., et al. (2000) Anastrozole versus Tamoxifen as First-Line Therapy for Advanced Breast Cancer in 668 Postmenopausal Women: Results of the Tamoxifen or Arimidex Randomized Group Efficacy and Tolerability Study. Journal of Clinical Oncology, 18, 3748-3757. https://doi.org/10.1200/JCO.2000.18.22.3748

[21] Mouridsen, H., Gershanovich, M., Sun, Y., Perez-Carrion, R., Boni, C., Monnier, A., et al. (2003) Phase III Study of Letrozole versus Tamoxifen as First-Line Therapy of Advanced Breast Cancer in Postmenopausal Women: Analysis of Survival and Update of Efficacy from the International Letrozole Breast Cancer Group. Journal of Clinical Oncology, 21, 2101-2109. https://doi.org/10.1200/JCO.2003.04.194

[22] Paridaens, R.J., Dirix, L.Y., Beex, L.V., Nooij, M., Cameron, D.A., Cufer, T., et al. (2008) Phase III Study Comparing Exemestane with Tamoxifen as First-Line Hormonal Treatment of Metastatic Breast Cancer in Postmenopausal Women: The European Organisation for Research and Treatment of Cancer Breast Cancer Cooperative Group. Journal of Clinical Oncology, 26, 4883-4890.

https://doi.org/10.1200/JCO.2007.14.4659

[23] Ellis, M.J., Llombart-Cussac, A., Feltl, D., Dewar, J.A., Jasiowka, M., Hewson, N., et al. (2015) Fulvestrant $500 \mathrm{mg}$ versus Anastrozole $1 \mathrm{mg}$ for the First-Line Treatment of Advanced Breast Cancer: Overall Survival Analysis from the Phase II FIRST Study. Journal of Clinical Oncology, 33, 3781-3787. https://doi.org/10.1200/JCO.2015.61.5831

[24] Robertson, J.F.R., Bondarenko, I.M., Trishkina, E., Dvorkin, M., Panasci, L., Manikhas, A., et al. (2016) Fulvestrant 500 mg versus Anastrozole $1 \mathrm{mg}$ for Hormone Receptor-Positive Advanced Breast Cancer (FALCON): An International, Randomised, Double-Blind, Phase 3 Trial. The Lancet, 388, 2997-3005. https://doi.org/10.1016/S0140-6736(16)32389-3

[25] Ali, S. and Coombes, R.C. (2002) Endocrine-Responsive Breast Cancer and Strategies for Combating Resistance. Nature Reviews Cancer, 2, 101-112. https://doi.org/10.1038/nrc721

[26] Osborne, C.K., Kitten, L. and Arteaga, C.L. (1989) Antagonism of Chemotherapy-Induced Cytotoxicity for Human Breast Cancer Cells by Antiestrogens. Journal of Clinical Oncology, 7, 710-717.

[27] Kurebayashi, J., Nukatsuka, M., Sonoo, H., Uchida, J. and Kiniwa, M. (2010) Preclinical Rationale for Combined Use of Endocrine Therapy and 5-Fluorouracil But Neither Doxorubicin Nor Paclitaxel in the Treatment of Endocrine-Responsive Breast Cancer. Cancer Chemotherapy and Pharmacology, 65, 219-225. https://doi.org/10.1007/s00280-009-1024-7 
[28] Goldenberg, G.J. and Froese, E.K. (1985) Antagonism of the Cytocidal Activity and Uptake of Melphalan by Tamoxifen in Human Breast Cancer Cells in Vitro. Biochemical Pharmacology, 34, 763-770. https://doi.org/10.1016/0006-2952(85)90755-5

[29] Nukatsuka, M., Saito, H., Nakagawa, F., Abe, M., Uchida, J., Shibata, J., et al. (2011) Oral Fluoropyrimidine May Augment the Efficacy of Aromatase Inhibitor via the Down-Regulation of Estrogen Receptor in Estrogen-Responsive Breast Cancer Xenografts. Breast Cancer Research and Treatment, 128, 381-390.

https://doi.org/10.1007/s10549-010-1141-3

[30] Rivkin, S.E., Green, S., Metch, B., Cruz, A.B., Abeloff, M.D., Jewell, W.R., et al. (1994) Adjuvant CMFVP versus Tamoxifen versus Concurrent CMFVP and Tamoxifen for Postmenopausal, Node-Positive, and Estrogen Receptor-Positive Breast Cancer Patients: A Southwest Oncology Group Study. Journal of Clinical Oncology, 12, 2078-2085. https://doi.org/10.1200/JCO.1994.12.10.2078

[31] Bottini, A., Generali, D., Brizzi, M.P., Fox, S.B., Bersiga, A., Bonardi, S., et al. (2006) Randomized Phase II Trial of Letrozole and Letrozole plus Low-Dose Metronomic Oral Cyclophosphamide as Primary Systemic Treatment in Elderly Breast Cancer Patients. Journal of Clinical Oncology, 24, 3623-3628. https://doi.org/10.1200/JCO.2005.04.5773

[32] Anders, C.K., Hsu, D.S., Broadwater, G., Acharya, C.R., Foekens, J.A., Zhang, Y., et al. (2008) Young Age at Diagnosis Correlates with Worse Prognosis and Defines a Subset of Breast Cancers with Shared Patterns of Gene Expression. Journal of Clinical Oncology, 26, 3324-3330. https://doi.org/10.1200/JCO.2007.14.2471

[33] Chang, J., Clark, G.M., Allred, D.C., Mohsin, S., Chamness, G. and Elledge, R.M. (2003) Survival of Patients with Metastatic Breast Carcinoma: Importance of Prognostic Markers of the Primary Tumor. Cancer, 97, 545-553. https://doi.org/10.1002/cncr.11083

[34] Lonning, P.E., Bajetta, E., Murray, R., Tubiana-Hulin, M., Eisenberg, P.D., Mickiewicz, E., et al. (2000) Activity of Exemestane in Metastatic Breast Cancer after Failure of Nonsteroidal Aromatase Inhibitors: A Phase II Trial. Journal of Clinical Oncology, 18, 2234-2244. https://doi.org/10.1200/JCO.2000.18.11.2234 